\title{
Conceptual design of controllers for automated modular construction machines
}

\author{
Edgar C. Tamayo ${ }^{\mathrm{a}^{*}}$, Ahmed Jawad Qureshi ${ }^{\mathrm{a}}$, Petr Musilek ${ }^{\mathrm{a}}$, Mohamad Al-Hussein ${ }^{\mathrm{a}}$ \\ ${ }^{a}$ University of Alberta, Edmonton T6G 1H9, Canada
}

\begin{abstract}
Without a methodology, the practice of control system design for automated modular construction machines mainly depends on experience and trial-and-error. Implementation of controllers requires planning at the conceptual design phase. Axiomatic design (AD) has been introduced in developing control solutions. This paper formalizes the conceptual design methodology in building a controller with the use of quality function deployment (QFD) as a design and an analysis tool. The controller design approach using QFD has been applied to the automated steel wall framing machine and to a 2-degree-of-freedom (DOF) robotic arm, which can be readily extended to $n$-DOF robotic manipulators. The analysis and decoupling techniques for controller design presented in this paper differ from those used in traditional AD. QFD for controller design provides continuous transfer functions to represent relationships and mathematical decoupling that is easily implemented in software.
\end{abstract}

(C) 2019 The Authors. Published by Budapest University of Technology and Economics \& Diamond Congress Ltd. Peer-review under responsibility of the scientific committee of the Creative Construction Conference 2019.

Keywords: controller design; decoupling; modular construction; quality function deployment; robotic manipulators

\section{Introduction}

Automation in construction arises due to the performance limitations of conventional construction [1] (Systems that realize the automation are, however, complex. An automated steel wall framing machine [2], for example, consists of mechanical, electrical, and control systems. Difficulties associated with designing an automated system can be overcome by the use of model-based system engineering (MBSE) methodology [3]. MBSE methodologies can be categorized as graphical or matrix-based. When adopted during the conceptual design phase, the matrix-based methodology provides a visual, compact, systematic, and transdisciplinary integrated MBSE approach [2].

To correct the usual practices in machine control system design that depend on experience and trial-and-error, Lee et al. [4] have illustrated the applicability of axiomatic design in developing control solutions. These authors have not employed QFD and process control decoupling techniques. QFD is a tool to align product design with customer needs [5]. A sequence of QFDs has been applied from customer needs identification, product planning, part planning, process planning and production control [6], which depicts the usefulness of QFD as a design and an analysis tool. Although not specifically called QFD, Lahiri [7] has applied the methodology only to develop a step test plan in the form of an 
Tamayo, E. C., et al./ Proceedings of the Creative Construction Conference (2019) 004

https://doi.org/10.3311/CCC2019-004

expectation matrix for modelling a multivariable process. A formalized conceptual design methodology is still required in designing a controller.

This paper focuses on the use of the QFD matrix at the AD and DSM stages of the integrated conceptual design MBSE approach and is structured as follows. Section 2 describes the QFD matrix for controller design. Section 3 illustrates the applications of the controller design methodology. A discussion of results is discussed in Section 4 followed by the conclusion in Section 5.

\section{Conceptual design of controllers}

An integrated MBSE methodology involves the repeated use of quality function model (QFD) throughout the stages of conceptual design, namely: (a) customer requirements definition; and (b) integrate function modelling (IFM) development with the use of axiomatic design (AD) and design structure matrix (DSM). Fig. 1 depicts the multiple applications of QFD, which establishes a model-based conceptual design approach. In this figure, the controller design extracted from AD and DSM is represented as a QFD matrix, in P-canonical or V-canonical form, that dynamically relates the manipulated variables (MV) and control variables (CV) through continuous or discrete transfer functions. Two QFD matrices are required to fully represent a V-canonical structure and to properly apply a corresponding decoupling strategy. In following the traditional representation, it could have been more appropriate to show only one QFD with its roof comprising the CV correlation matrix. However, two matrices are used to emphasize the AD and DSM steps in the conceptual design of a controller as well. This QFD representation in the design of multivariable predictive controllers is called expectation matrix [7]. Inside the IFM is a collection of QFD matrices that constitutes the state, process/use case, actors, and interaction views. For the electrical control panel, the conceptual design method introduced in a study by Tamayo et al. [8] facilitates the subsequent computer-aided design to be performed at the detailed design stage. Controller QFD will be shown to have numeric, Boolean, and transfer function representations depending on the type of systems being developed.

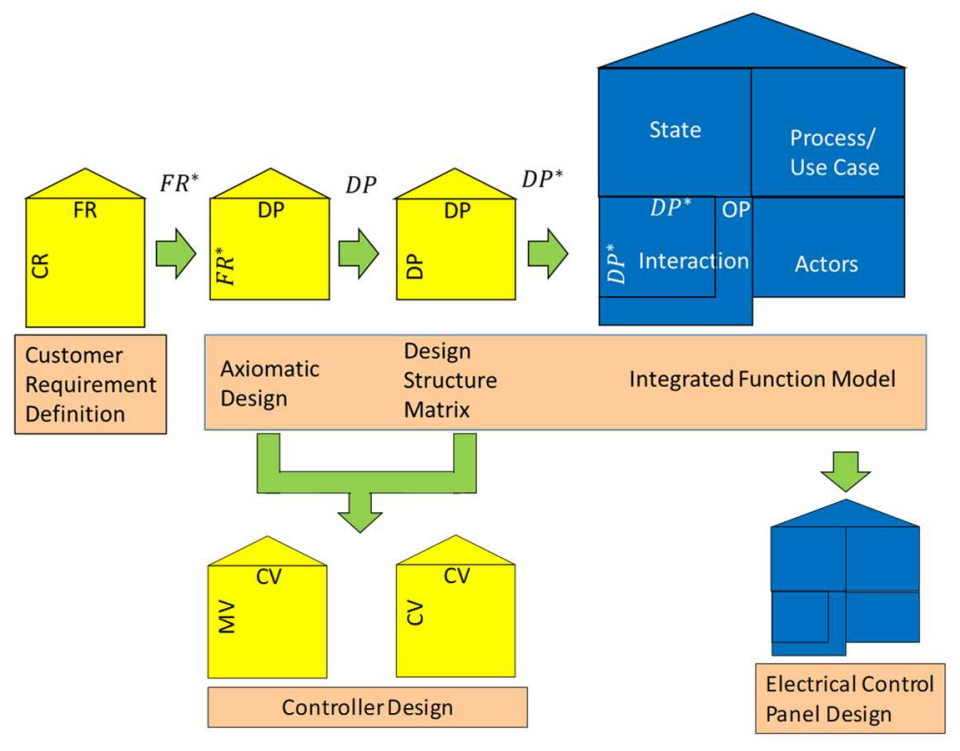

Fig. 1. Integrated conceptual design overview

\subsection{QFD structures for controller design}

As depicted in Fig. 1, a QFD matrix for controller design is extracted from the overall design matrix (DM) and DSM for the following reasons: (i) to express interactions in Laplace transfer functions; (ii) to identify coupled interactions; (iii) to design decouplers in enabling the designers to treat the control problem as loops consisting of independent 
Tamayo, E. C., et al./ Proceedings of the Creative Construction Conference (2019) 004 https://doi.org/10.3311/CCC2019-004

systems; and (iv) to facilitate simulation at the conceptual design phase. Although controllers are not implemented in Laplace transfer functions, this QFD representation effectively communicates the behavior of the multivariable process.

Fig. 2 illustrates a $2 \times 2$ multivariable process with its corresponding QFD matrix in P-canonical and V-canonical forms [9]. As in AD, coupling is shown as the off-diagonal elements of the matrix in terms of Laplace transfer functions. A completely decoupled system is desired since it allows designers to implement multiple single-input-single-output (SISO) controllers. A decoupling strategy that is appropriate for each canonical structure is discussed further in the next section.
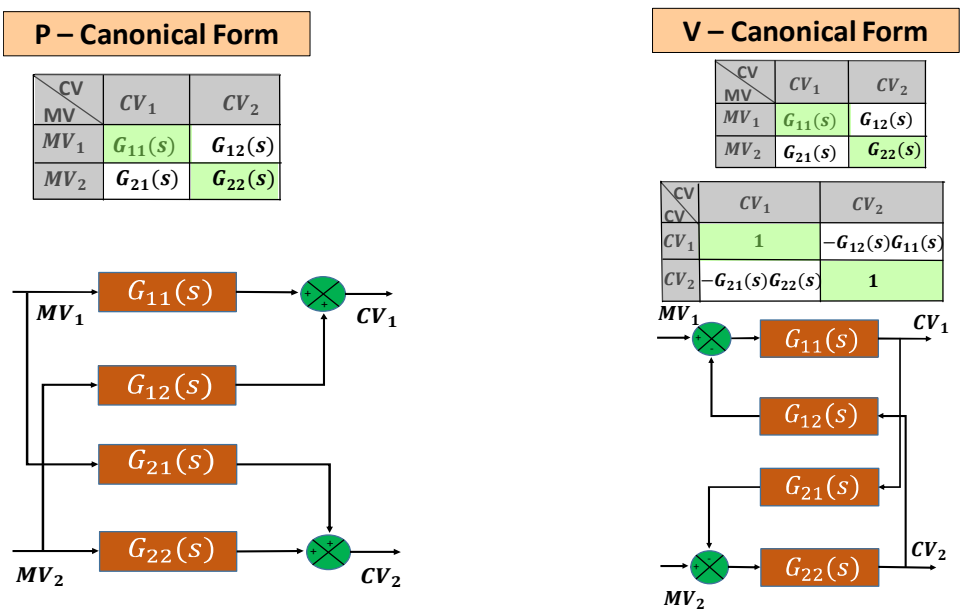

Fig. 2. QFD representations of a coupled $2 \times 2$ multivariable system

\subsection{Decoupling strategy for P-canonical form}

In this section, the decoupling techniques derived for $2 \times 2$ multivariable systems are easily extended to $n \times n$ multiple-input-multiple-output (MIMO) systems. It must be noted that a V-canonical form has an equivalent higher order P-canonical structure [9]. For simplicity, a V-canonical form is considered separately to avoid dealing with higher order transfer functions. A MIMO system of the P-canonical structure is expressed in the following equations:

$$
\begin{aligned}
& C V_{1}(s)=G_{11}(s) M V_{1}(s)+G_{12}(s) M V_{2}(s) \\
& C V_{2}(s)=G_{22}(s) M V_{2}(s)+G_{21}(s) M V_{1}(s)
\end{aligned}
$$

Fig. 3 depicts a decoupling technique, with multi-loop PID feedback controllers, for the P-canonical structure that implements a diagonalization process in the following equation:

$$
Q(s) G(s)=W(s)
$$

where the decoupling transfer matrix, $Q(s)$, is chosen such that $W(s)$ is diagonal. Eq. 1 has multiple solutions, but the simplest solution takes $Q_{11}(S)=Q_{22}(S)=1$. In this case, $Q_{12}(S)$ and $Q_{21}(S)$ are then determined as follows (Marlin 2015):

$$
\begin{aligned}
& Q_{12}(s)=-\frac{G_{12}(s)}{G_{11}(s)} \\
& Q_{21}(s)=-\frac{G_{21}(s)}{G_{22}(s)}
\end{aligned}
$$


Tamayo, E. C., et al./ Proceedings of the Creative Construction Conference (2019) 004 https://doi.org/10.3311/CCC2019-004

Thus, for the general MIMO case, the decoupling transfer matrix can be expressed as follows:

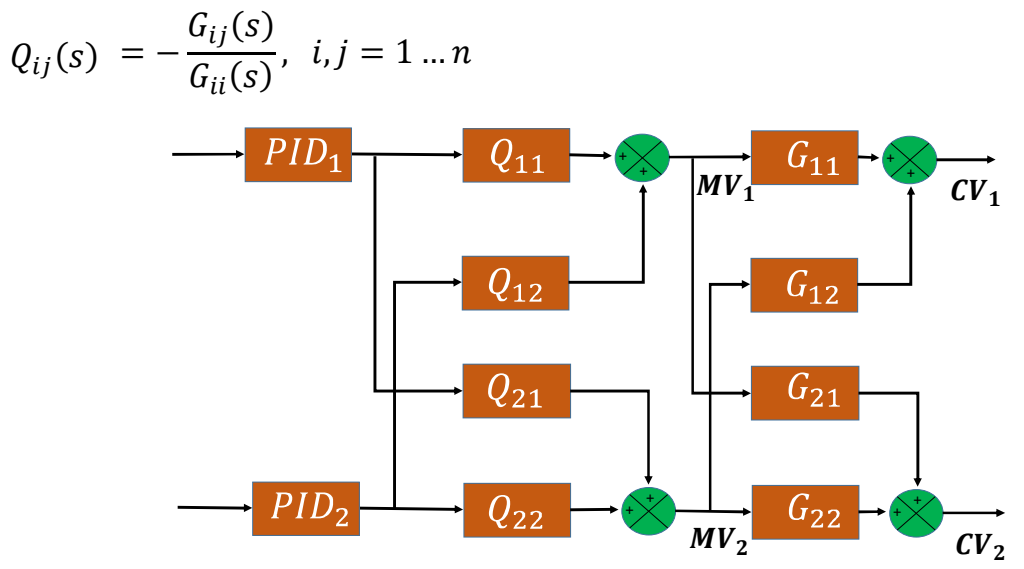

Fig. 3. Decoupling technique for a P-canonical MIMO system with PID controllers

\subsection{Decoupling strategy for $V$-canonical form}

A V-canonical structure is encountered in MIMO processes involving the following model, where the transfer function matrix, $G(s) \in C^{n \times n}$ :

$$
C V(s)=G(s) M V(s)
$$

A model reference approach to decoupling requires that the output follow the reference command signal, which implies obtaining a feedforward controller, $Q(s)=G(s)^{-1}$, since

$$
\begin{aligned}
C V(s) & =Q(s) G(s) C V_{\text {ref }}(s) \\
& =C V_{\text {ref }}(s)
\end{aligned}
$$

Eq. 9 defines a feedforward controller that is the inverse of the process. This observation suggests using the linear or non-linear dynamic model of the process for decoupling by simply feeding the appropriate reference signals to the feedforward controller and augmenting its output to that of the feedback controller.

Thus, for the $2 \times 2$ MIMO system in Fig. 4, the feedforward controllers are:

$$
\begin{aligned}
& Q_{1}(s)=C V_{1 r e f}(s) G_{11}(s)^{-1}+G_{12}(s) C V_{2 r e f}(s) \\
& Q_{2}(s)=C V_{1 r e f}(s) G_{22}(s)^{-1}+G_{21}(s) C V_{2 r e f}(s)
\end{aligned}
$$




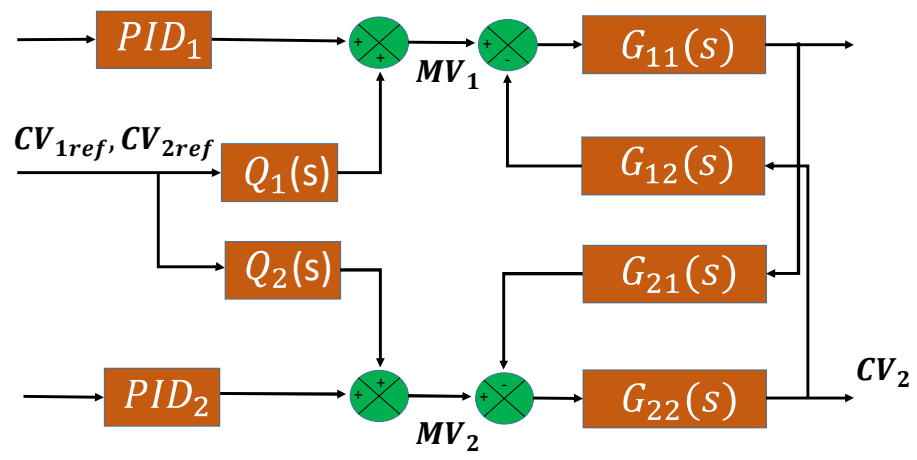

Fig. 4. Decoupling technique for a V-canonical MIMO system with PID controllers

\section{Illustrative examples}

\subsection{Steel wall framing machine}

An automated steel wall framing machine is illustrated in Fig. 5. This machine is capable of making three types of wall panels, namely: (i) with studs only; (ii) with studs and window; and (iii) with studs and door. Screw fastening is accomplished by first manually preparing the frames for automatic fastening, then entering the appropriate database for the frame being manufactured, and finally initiating the automatic screw fastening process.

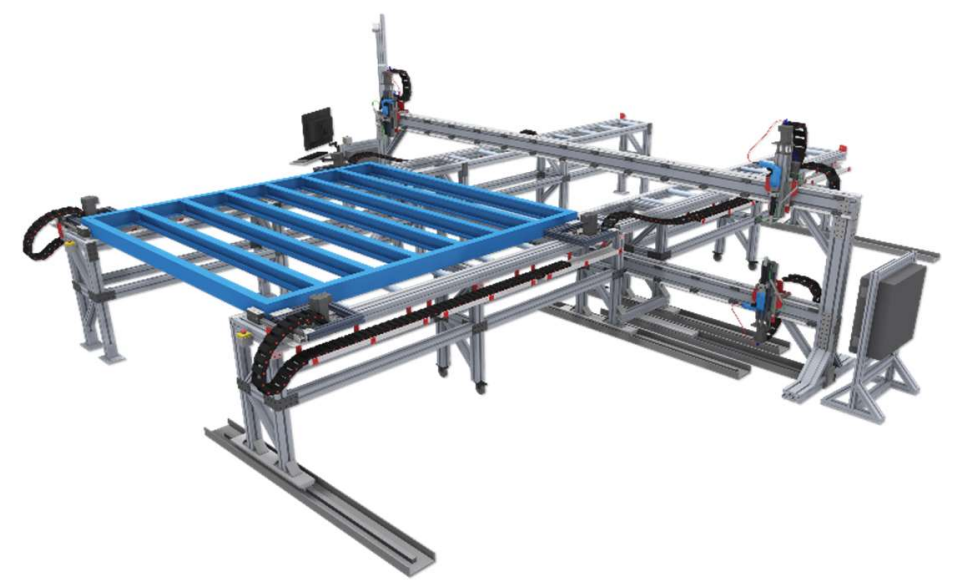

Fig. 5. Automated steel wall framing machine

Features of the machine includes two power screwdrivers on the top gantry and two on the bottom gantry. To accommodate different widths of wall panels, one side of the table is positioned accordingly. Each dragging device is positioned at a right angle to ensure that the panel is square. Thus, the QFD matrix for control design depicted in Table 1 is developed using the functional requirements (FRs) extracted at the AD phase of the integrated conceptual design, and then observing that none of the motors are linked. Since movements are accomplished using stepper motors, the MVs and CVs in the QFD table are torques and distances, respectively. 
Tamayo, E. C., et al./ Proceedings of the Creative Construction Conference (2019) 004 https://doi.org/10.3311/CCC2019-004

Table 1. QFD matrix for the steel wall framing machine controllers

\begin{tabular}{llc|c|c|ccc}
\hline & & $C V_{1}$ & $C V_{2}$ & $C V_{3}$ & $C V_{4}$ & $C V_{5}$ & $C V_{6}$ \\
\hline Table & $M V_{1}$ & $\mathrm{X}$ & & & & & \\
Clamp & $M V_{2}$ & & $\mathrm{X}$ & & & & \\
Dragging & $M V_{3}$ & & $\mathrm{X}$ & & & \\
Y-axis position & $M V_{4}$ & & & $\mathrm{X}$ & & \\
Z-axis position & $M V_{5}$ & & & & $\mathrm{X}$ & \\
Fastening & $M V_{6}$ & & & & & $\mathrm{X}$ \\
\end{tabular}

It is clear from the QFD matrix that the positioning control systems are uncoupled, indicating that each motor can be controlled individually, and that closed-loop stepper motors can be considered to simplify controller implementation.

\subsection{Two link planar robotic arm}

3.3. Robotic manipulators are coupled since the angular position of a motor in a joint affects those in the other joints. To illustrate the use of the QFD controller design described in the previous sections, a 2-DOF PUMA 560 robotic arm [10] is then discussed.

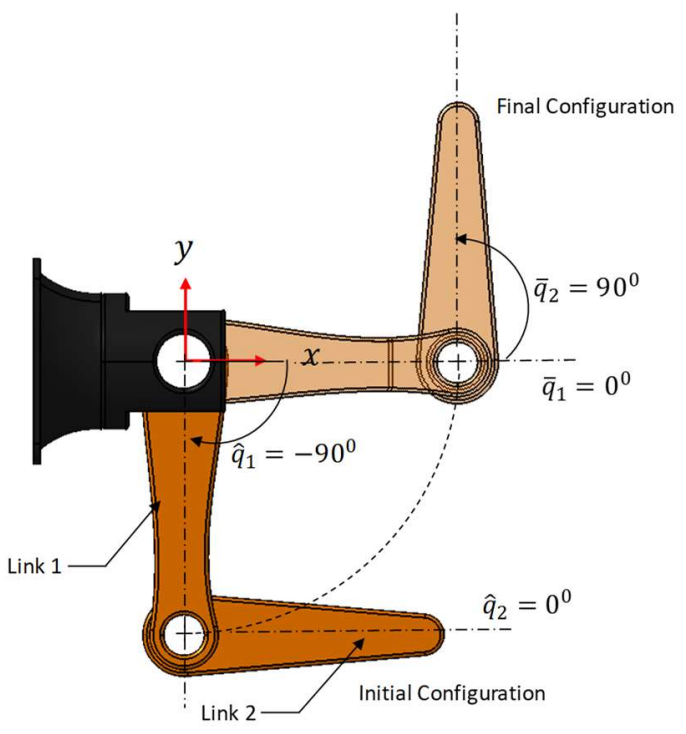

Fig. 6. 2-DOF robotic arm

Torque, $\tau \in R^{2}$, is dynamically related to the joint angle, $q \in R^{2}$, for the 2-DOF robotic arm shown in Fig. 6 as follows (Craig 2005):

$$
\tau=M(q) \ddot{q}+V(q, \dot{q})+G(q)
$$

where for the masses and lengths of the first and second link as respectively $m_{1}, m_{2}, l_{1}$, and $l_{2}$, the following mass matrix, $M(q)$, centrifugal and Coriolis matrix, $V(q, \dot{q})$, and gravity matrix, $G(q)$, are obtained.

$$
M(q)=\left[\begin{array}{cc}
l_{2}{ }^{2} m_{2}{ }^{2}+2 l_{1} l_{2} m_{2} \cos q_{2}+l_{1}{ }^{2}\left(m_{1}+m_{2}\right) & l_{2}{ }^{2} m_{2}{ }^{2}+l_{1} l_{2} m_{2} \cos q_{2} \\
l_{2}{ }^{2} m_{2}{ }^{2}+l_{1} l_{2} m_{2} \cos q_{2} & l_{2}{ }^{2} m_{2}{ }^{2}
\end{array}\right]
$$


Tamayo, E. C., et al./ Proceedings of the Creative Construction Conference (2019) 004 https://doi.org/10.3311/CCC2019-004

$$
\begin{aligned}
V(q, \dot{q}) & =\left[\begin{array}{c}
-m_{2} l_{1} l_{2} \sin q_{2}{\dot{q_{2}}}^{2}-2 l_{1} l_{2} m_{2} \sin q_{2} \ddot{q}_{2} \\
l_{1} l_{2} m_{2} \sin q_{2} \dot{q}_{1}{ }^{2}
\end{array}\right] \\
G(q) & =\left[\begin{array}{c}
m_{2} l_{2} g \cos \left(q_{1}+q_{2}\right)+\left(m_{1}+m_{2}\right) l_{1} g \cos q_{1} \\
m_{2} l_{2} g \cos \left(q_{1}+q_{2}\right)
\end{array}\right]
\end{aligned}
$$

Using the example in (Seraji 1987), Eq. 7 is linearized at the operating point $\left[\bar{q}_{1}, \overline{\dot{q}}_{1} ; \bar{q}_{2}, \overline{\dot{q}}_{2}\right]=\left[-\frac{\pi}{2}, 0 ; 0,0\right]$ to obtain the following equation.

$$
\tau=A \ddot{q}+B \dot{q}+C q
$$

For the PUMA example with model parameters $a_{1}=3.82, a_{2}=2.12, a_{3}=0.71, a_{4}=81.82$, and $a_{5}=24.06$,

$$
\begin{aligned}
& A=\left[\begin{array}{cc}
a_{1}+a_{2} \cos \bar{q}_{2}+l_{1}{ }^{2}\left(m_{1}+m_{2}\right) ; & a_{3}+0.5 a_{2} \cos \bar{q}_{2} \\
a_{3}+0.5 l_{2} \cos \bar{q}_{2} & a_{3}
\end{array}\right] \\
& B=\left[\begin{array}{cc}
-a_{2} \overline{\dot{q}}_{2} \sin \bar{q}_{2} ; & -a_{2}\left(\overline{\dot{q}}_{1}+\overline{\dot{q}}_{2}\right) \sin \bar{q}_{2} \\
a_{2} \overline{\dot{q}}_{2} \sin \bar{q}_{2} & 0
\end{array}\right] \\
& C=\left[\begin{array}{cc}
-a_{4} \sin \bar{q}_{1}-a_{5} \sin \left(\bar{q}_{1}+\bar{q}_{2}\right) ; & -a_{5} \sin \left(\bar{q}_{1}+\bar{q}_{2}\right)-a_{2}\left(\overline{\dot{q}}_{1} \overline{\dot{q}}_{2}+0.5 \overline{\dot{q}}_{2}^{2}\right) \cos \bar{q}_{2} \\
-a_{5} \sin \left(\bar{q}_{1}+\bar{q}_{2}\right) & -a_{5} \sin \left(\bar{q}_{1}+\bar{q}_{2}\right)+0.5 a_{2} \cos \bar{q}_{2}
\end{array}\right]
\end{aligned}
$$

Taking the Laplace transform of Eq. 15 for the $2 \times 2$ robotic arm and noting that $\tau=M V$ and $q=C V$, the result is the following equation.

$$
M V=\left(A s^{2}+B s+C\right) C V
$$

\begin{tabular}{|c|c|c|}
\hline CV & $C V_{1}$ & $C V_{2}$ \\
\hline$M V_{1}$ & $G_{11}=\frac{1}{A_{11} s^{2}+B_{11} s+C_{11}}$ & $G_{12}=A_{12} s^{2}+B_{12} s+C_{12}$ \\
\hline$M V_{2}$ & $G_{21}=A_{21} s^{2}+B_{21} s+C_{21}$ & $G_{22}=\frac{1}{A_{22} S^{2}+B_{22} s+C_{22}}$ \\
\hline $\mathrm{CV}$ & $C V_{1}$ & $C V_{2}$ \\
\hline$C V_{1}$ & 1 & $-\frac{A_{12} s^{2}+B_{12} s+C_{12}}{A_{11} s^{2}+B_{11} s+C_{11}}$ \\
\hline$C V_{2}$ & $-\frac{A_{21} s^{2}+B_{21} s+C_{21}}{A_{22} s^{2}+B_{22} s+C_{22}}$ & 1 \\
\hline
\end{tabular}

or

$\left[\begin{array}{l}M V_{1} \\ M V_{2}\end{array}\right]=\left\{\left[\begin{array}{ll}A_{11} & A_{12} \\ A_{21} & A_{22}\end{array}\right] s^{2}+\left[\begin{array}{ll}B_{11} & B_{12} \\ B_{21} & B_{22}\end{array}\right] s+\left[\begin{array}{ll}C_{11} & C_{12} \\ C_{21} & C_{22}\end{array}\right]\right\}\left[\begin{array}{l}C V_{1} \\ C V_{2}\end{array}\right]$

Eq. 19 reduces to Eq. 7 if $G(s)$ is taken to be equal to $\left(A s^{2}+B s+C\right)^{-1}$, therefore $Q(s)=\left(A s^{2}+B s+C\right)$. Applying the derivations for the feedforward controller of the $2 \times 2$ MIMO system leads to the following QFD matrix that utilizes the AD and DSM sections to fully describe the V-canonical structure in Fig. 7.

Fig. 7. QFD matrix of the 2-DOF PUMA 560 robot 
Tamayo, E. C., et al./ Proceedings of the Creative Construction Conference (2019) 004 https://doi.org/10.3311/CCC2019-004

Using the information provided in the QFD table, the feedforward controller for each loop is obtained as follows:

$$
\begin{aligned}
& Q_{1}(s)=\left(A_{11} s^{2}+B_{11} s+C_{11}\right) C V_{1 r e f}+\left(A_{12} s^{2}+B_{12} s+C_{12}\right) C V_{2 r e f} \\
& Q_{2}(s)=\left(A_{22} s^{2}+B_{22} s+C_{22}\right) C V_{1 r e f}+\left(A_{21} s^{2}+B_{21} s+C_{21}\right) C V_{2 r e f}
\end{aligned}
$$

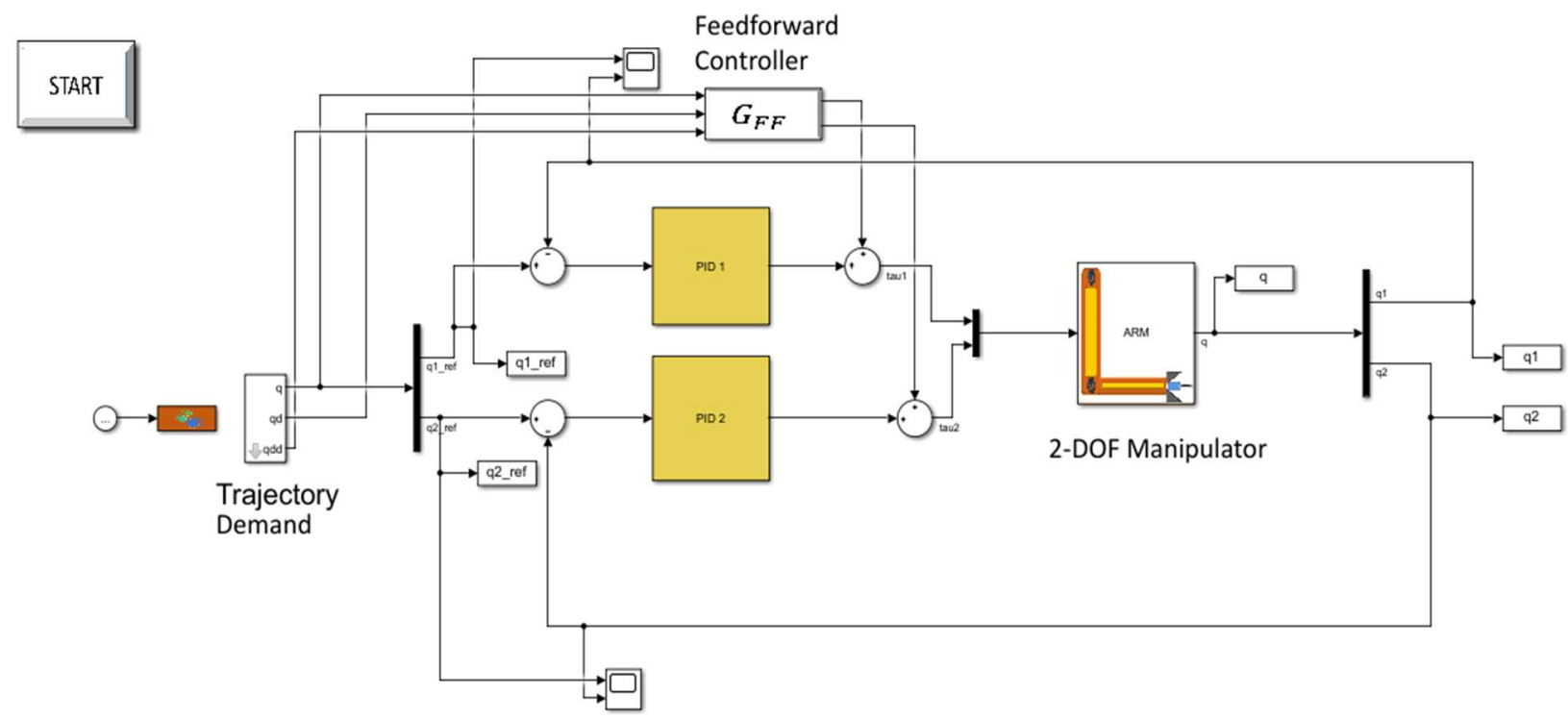

Fig. 8. Simulink control implementation of the 2-DOF robotic arm for an initial and final trajectory configuration of $\left[\bar{q}_{1}, \overline{\dot{q}}_{1} ; \bar{q}_{2}, \overline{\dot{q}}_{2}\right]_{i}=\left[-\frac{\pi}{2}, 0 ; 0,0\right]$ and $\left[\bar{q}_{1}, \overline{\dot{q}}_{1} ; \bar{q}_{2}, \overline{\dot{q}}_{2}\right]_{f}=\left[0,0 ; \frac{\pi}{2}, 0\right]$

Fig. 8 shows the Simulink model that implements the feedforward controller, $G_{F F}$, defined in Eqs. 13 and 14 and the PID controllers shown in Fig. 4 in controlling a nonlinear 2-DOF robotic arm. Fig. 9 shows the joint angular responses of the robotic arm to the trajectory configuration described in Fig. 6.
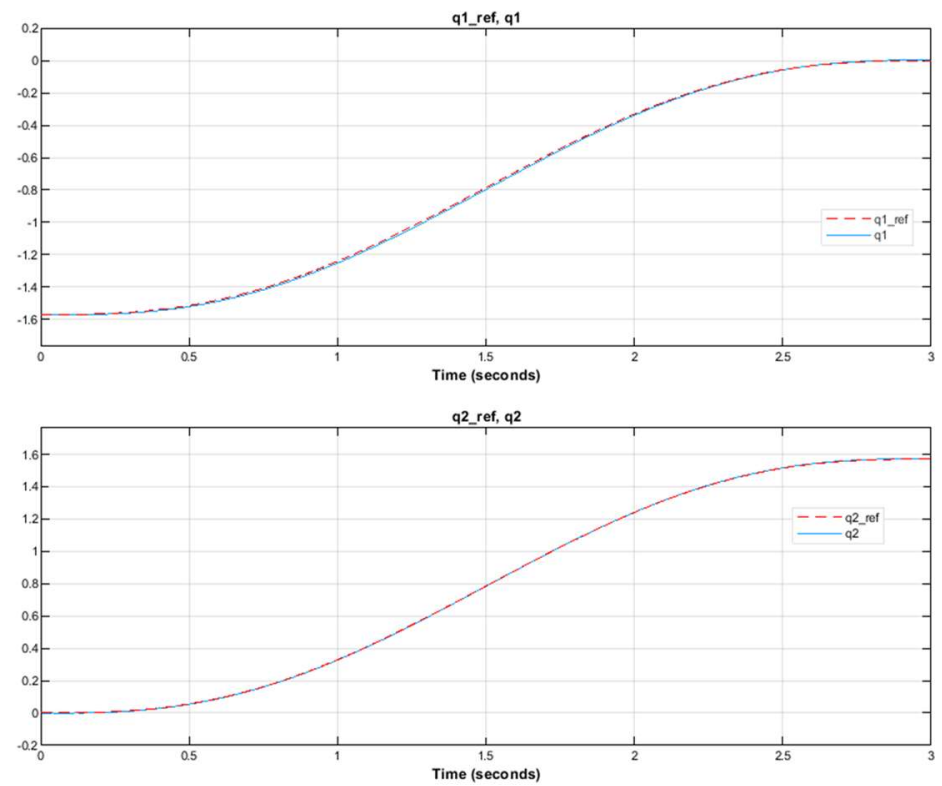

Fig. 9: Joint angle responses to the trajectory demand configuration of Fig. 6 
Tamayo, E. C., et al./ Proceedings of the Creative Construction Conference (2019) 004

https://doi.org/10.3311/CCC2019-004

\section{Conclusion}

A methodology for designing controllers at the conceptual design phase has been developed using QFD matrices. These control QFDs are extracted from the AD and DSM phases of integrated conceptual design. QFD, as a modelbased approach to controller design, is a thought process that encourages creativity, collaboration, and communication as illustrated in the systematic representation and analysis of the dynamic structure of MIMO processes and their associated decoupling solutions in this paper.

When adopted in conceptual design, the matrix-based methodology provides a visual, compact, systematic, and transdisciplinary integrated MBSE approach [2]. It has been demonstrated that the structure of the control QFD matrix or matrices corresponds to a particular control strategy to adopt, signifying that the proposed methodology is indeed systematic. Although the emphasis on decoupling a QFD matrix limits the strategy to linear decoupling, the framework developed in this paper can be extended to incorporate nonlinear feedforward and adaptive linear feedforward strategies for future work.

\section{References}

[1] T. Bock, "The future of construction automation: Technological disruption and the upcoming ubiquity of robotics," Autom. Constr., vol. 59, pp. 113-121, 2015. https://doi.org/10.1016/j.autcon.2015.07.022

[2] E. Tamayo, M. Bardwell, A. Qureshi, and M. Al-Hussein, "Automation of a steel wall framing assembly," in ISEC 2017 - 9th International Structural Engineering and Construction Conference: Resilient Structures and Sustainable Construction, 2017. https://doi.org/10.14455/ISEC.res.2017.19

[3] M. A. Abdelrazek, A. T. El-sheikh, M. Zayan, and A. M. Elhady, "Systems Engineering Management Using Transdisciplinary Quality System Development Lifecycle Model," World Acad. Sci. Eng. Technol. Int. J. Aerosp. Mech. Eng., vol. 11, no. 1, pp. 115-125, 2017.

[4] K. D. Lee, N. P. Suh, and J.-H. Oh, "Axiomatic Design of Machine Control System," CIRP Ann. - Manuf. Technol., vol. 50, no. 1, pp. 109-114, 2001. http://dx.doi.org/10.1016/S0007-8506(07)62083-6

[5] F. Franceschini, Advanced Quality Function Quality Function Deployment. Boca Raton: CRC Press Company, 2002.

[6] G. Quesada and A. T. Bahill, "Improving Product Design Using Quality Function Deployment : The School Furniture Case in Developing Countries," vol. 16, no. 1, pp. 47-58, 2003. https://doi.org/10.1081/ QEN-120020770

[7] S. K. Lahiri, Multivariable Predictive Control, First Edit. New Jersey: John Wiley \& Sons, 2017.

[8] E. C. Tamayo, Y. I. Khan, A. J. Qureshi, and M. Al-Hussein, "Design automation of control panels for automated modular construction machines," Procedia CIRP, vol. 70, pp. 404-409, 2018. https://doi.org/10.1016/j.procir.2018.02.004

[9] M. T. Tham, "Multivariable Control: an Introduction To Decoupling Control †," Chem. Process Eng., no. July, pp. 1-19, 1999.

[10] H. Seraji, "An Approach to Multivariate Control of Manipulators," J. Dyn. Syst. Meas. Control, vol. 109, no. June 1987, pp. 146-54, 1987. http://dx.doi.org/10.1115/1.3143832 